\title{
L'AMOUR DANS L'OEUVRE D'EMMANUEL BOVE
}

Cyril Piroux, Université de Franche-Comté / Université du Québec à Chicoutimi

\section{Résumé analytique}

Quelle est la place accordée à l'amour dans l'œuvre d'Emmanuel Bove ? Interrogation paradoxale s'il en est car rien, a priori, ne laisserait entrevoir la possibilité de lier l'œuvre bovienne au thème du couple amoureux, tant la solitude, la misère et l'égoïsme étouffent ses personnages. Ceux-ci, cependant, pour pathétique que soit leur existence, aspirent sincèrement à aimer. Peut-être se trompent-ils simplement sur l'objet désiré. Un malentendu aux résonances proustiennes vouant chaque relation à la souffrance et à l'échec et qui pourtant mène à la connaissance de soi, l'intérêt du personnage bovien se centrant avant tout sur lui-même.

L'histoire littéraire a cette particularité à la fois désagréable et tout à fait passionnante de garder au fond d'elle-même quantité d'auteurs oubliés, comme un océan ses richesses. Emmanuel Bove (1898-1945) est l'un de ces trésors. Il fait partie de ces écrivains dont on se demande bien, après les avoir lus, comment on a pu les délaisser ainsi. Romancier, nouvelliste, journaliste, il fut l'une des révélations littéraires de l'entre-deux-guerres. À vingt-cinq ans seulement, il se fait connaître du public avec Mes Amis. Ce premier roman est accueilli avec enthousiasme par la critique. Très vite, la qualité de son style est reconnue par ses pairs. On le compare à Dostoïevski, à Proust. Les années suivantes, il confirme son talent en publiant trois autres romans dont La Coalition pour lequel, avec Mes Amis, il reçoit le Prix Figuière en 1928 devant André Malraux notamment, et Drieu la Rochelle. Sa capacité à survivre à l'effervescence intellectuelle des années folles aurait dû présager de sa longévité. Il n'en est rien. Le 14 juillet 1945 , tout juste âgé de 47 ans, il décède dans l'indifférence générale. Son œuvre, jugée trop noire et défaitiste à la Libération ne trouve plus sa place dans une littérature tournée vers l'engagement dès les années trente.

\section{Le(petit) manège amoureux}

La passion amoureuse n'a pas totalement déserté le roman bovien. De nombreux titres attestent d'ailleurs sa présence de manière explicite : L'Amour de Pierre Neuhart (1928), L'impossible amour (1935) ou sous la forme de dénominations implicites dénotant une vision particulièrement pessimiste de l'amour, comme Une fugue (1929), Une Illusion (1930), Un malentendu (1930), Un célibataire (1932)... Le premier roman d'Emmanuel Bove, Mes Amis, s'articule essentiellement autour du thème amoureux et des quatre femmes qui le constitue ${ }^{1}$. Pour chaque récit, une rencontre. Et pour chaque rencontre, l'apparition d'un personnage féminin, clé de voûte de la structure narrative. Bien sûr, la plupart de ces rencontres se termine en général par un échec retentissant. Les femmes s'enchaînent ainsi comme autant de figures symboliques de l'impossibilité d'aimer. Mais l'amour est là pourtant. Au centre de l'oeuvre. Au centre de l'écriture. Dans La Coalition (1928), I'histoire d'amour entre Nicolas Aftalion et Simone, si anecdotique qu'elle puisse paraître au regard de la déliquescence du jeune homme et de sa mère, est d'ailleurs placée au centre géographique de l'œuvre. Il n'est pas rare, en outre, au hasard d'un coup de foudre, que naisse le sentiment amoureux. Un certain romantisme d'avant-guerre s'exprime encore lors de ces rencontres inopinées. Mais il répond

1 François Ouellet, «La maîtrise implacable de l'écriture subjective », préface à l'édition de poche de Mes Amis, Nota Bene, 2002. 
toutefois à une sorte de schéma-type laissant présager un traitement de l'amour pour le moins parodique. Tout se fait alors selon les règles, d'après une structure narrative et émotionnelle bien définie, comme un cliché en somme, et avec une telle assiduité qu'on croit presque assister finalement à une sorte de parodie de Roméo et Juliette. Avant même le début de I'histoire, la monotonie des habitudes amoureuses prend le pas sur la spontanéité et l'authenticité des sentiments, d'emblée sclérosés par les dérives artificielles et hypocrites de la comédie humaine. Et l'amour devient un « calcul », une «comédie », un « petit manège ». Tout, en somme, semble joué d'avance, c'est le cas de le dire.

Le bilan des histoires d'amour sur l'ensemble des œuvres boviennes est à ce titre plutôt négatif. Mis à part dans L'Impossible amour, où les deux amants se retrouvent enfin après de multiples péripéties, l'ombre de la rupture plane sans éclaircie sur chaque relation. Armand, le personnage du roman éponyme (1927), est rejeté dans la misère d'où Jeanne l'avait extirpé quelques années auparavant. Les couples de La Coalition et de L'Amour de Pierre Neuhart finissent également par se séparer. Tout comme celui d'Un Caractère de femme (1936). Le lecteur n'est pas tenu très longtemps en haleine car il comprend rapidement que toute initiative - de séduction ou d'engagement amoureux - est en général vouée à l'échec. Les seules expériences amoureuses accessibles à Victor Bâton, l'anti-héros de Mes Amis, se limitent en soi à quelques relations stables et durables d'un soir après lesquelles, en général, chacun retourne à ses occupations, comme si rien ne s'était passé2.

Comment juger également à la pérennité du couple quand les personnages doutent de leurs propres chances ? La plupart du temps, en effet, les prétendants n'y croient pas eux-mêmes, comme si un pressentiment tragique les cloîtrait dans une sorte de résignation fataliste et de méfiance à l'égard des femmes. Lors de sa promenade avec Blanche de Myrtha, Victor Bâton déclare : «J'aurais été tout à fait heureux si je n'avais pas appréhendé une bizarrerie subite. Les femmes sont si étranges. Ma voisine était capable, tout à coup, au coin d'une rue, de me dire au revoir. ${ }^{3} \gg$ Quelques lignes et mètres plus tard, il reprend :

J'étais heureux. Pourtant, des réflexions ridicules gâtaient ma joie.

Qu'aurait fait Blanche, si nous avions rencontré sa meilleure amie ? M'eût-elle quitté ? Ou bien si, tout à coup, une douleur l'eût empêché de marcher ? Ou bien encore, si elle avait cassé une vitre, ou déchiré sa robe, ou bousculé un passant ?

Je me demande parfois si je ne suis pas fou. J'avais tout pour être heureux et il fallait que des réflexions idiotes vinssent me troubler ${ }^{4}$.

Ce type de lamentations pathétiques révèle au lecteur le caractère mal assuré des personnages masculins qui semblent bien incapables d'aimer. Ces derniers ne peuvent s'empêcher de se compliquer la vie, de tout gâcher, comme si l'idée même d'être heureux les angoissait. Cela expliquerait pourquoi, chez Bove, les scènes de séparation sont rarement violentes. Les protagonistes acceptent leur sort sans lutter, sans éclat. Aucun cri, aucun pleur. Pas de supplication. L'échec, indéniablement, est inhérent à l'amour. C'est une fatalité, une normalité. Et du même coup, l'occasion de recommencer autre chose, ailleurs, l'occasion d'aller à l'aventure, plutôt qu'à l'amour.

Bove dénonce sans ménagement ou presque le couple dans tout ce qu'il a de plus hypocrite : « Les amoureux

2 C'est le cas par deux fois avec Lucie Dunois puis Blanche de Myrtha.

3 Emmanuel Bove, Mes Amis, Flammarion, 2006, p. 117.

4 Mes Amis, opus cit., p. 118. 
sont égoïstes et impolis ${ }^{5} »$, s'écrie Victor Bâton, avant de se contredire quelques pages plus loin : «Je veux croire qu'un jour je serai heureux, qu'un jour quelqu'un m'aimera ${ }^{6} »$. L'auteur de Mes Amis, cependant, est généreux et cette déstructuration du couple, toute cruelle qu'elle puisse paraître, se fait avec le charme de la dérision. Nous sommes bien loin ici, de l'acidité de Céline pour qui l'amour serait «l'infini mis à la portée des caniches ». La critique bovienne de l'amour est moins cynique et tout aussi efficace peut-être. Comment ne pas sourire ainsi au comique de situation, aux descriptions fantasques et aux scènes ridicules durant lesquelles les personnages perdent toute constance. Ainsi en est-il par exemple dans Mes Amis, lors de la scène de baiser entre Victor Bâton et Blanche de Myrtha.

[...] je ne songeai plus qu'à l'embrasser, mais je n'osais pas en marchant, de crainte de manquer la bouche.

- Arrêtons-nous. Je voudrais vous dire quelque chose.

Ma voix tremblait. Je pris ses mains et lissait mes lèvres avec les dents.

- Que vouliez-vous me dire, monsieur?

Je la serrai contre moi. Nos genoux se cognèrent comme des boules de bois. Je fis attention à ne pas perdre l'équilibre pour ne pas lui marcher sur les pieds.

Puis, subitement, je l'embrassai.

En me redressant, je sentis que mon chapeau déplaçait le sien.

Bien qu'elle le remît rapidement sur les yeux, je sentis que cela l'avait gênée.

Penaud, les bras ballants, je ne savais si je devais de nouveau embrasser ma compagne ou bien m'excuser 7 .

Il y a quelque chose de "visuel" dans cet extrait qui relève presque autant semble-t-il de la littérature que du cinéma muet à la Mack Sennett par exemple. Bove écrit comme il voit. Les étapes du baiser s'enchaînent dans l'esprit de Bâton comme autant de plans donnant à la scène un aspect clairement séquentiel et rythmé. Par une succession d'effets comiques inattendus, rappelant les gags de Buster Keaton ou de Charlie Chaplin, Bove parvient à représenter avec humour la réalité parfois absurde et chaotique des relations amoureuses ${ }^{8}$. Aucun panache, aucune passion dans ce passage assez peu "glamour" il faut bien le dire. Les personnages masculins semblent frappés d'impuissance. Complètement dévirilisés. Au moment où il va embrasser sa compagne, Victor Bâton est obnubilé par un lot de préoccupations pratiques qui parasitent l'acte amoureux et finissent par rompre le charme. La scène est drôle, elle est grotesque. Mais elle manque cruellement de romantisme. On sourit des indélicatesses de Bâton et de sa roublardise, on s'afflige de tant de maladresses. Mais on ne rêve pas, assurément, devant cette expérience pour le moins décevante. Le moins que l'on puisse dire, c'est que le mythe de l'amour idéal est sévèrement mis à mal lors de telles

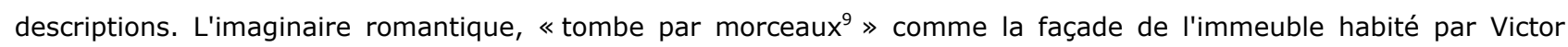
Bâton. Dans ses œuvres, Bove gratte avec subtilité les dernières couches de vernis recouvrant le visage éreinté du romantisme d'avant-guerre, que Proust et Huysmans avant lui avait déjà bien découvert. Ses personnages seraient ainsi en quelque sorte les porteurs d'un romantisme désenchanté ; un romantisme d'après-guerre, victime de son époque si l'on peut dire, sans fard et dévoilant une réalité du couple certes déceptive, mais bien réelle. D'un coup, la solution à l'une des plus grandes énigmes de la littérature et de l'homme en général nous apparaît avec force. Et le

5 Mes Amis, opus cit., p. 51.

6 Ibid., p. 125.

7 Ibid., p. 118.

8 Notons-le, l'aspect "visuel" de l'écriture bovienne est renforcé, nous avons pu le constater lors du colloque, par l'effet comique que produit sa lecture. Il y a chez Bove un humour qui contraste de manière singulière avec le pessimisme inhérent à son oeuvre. Et cet humour est si particulier qu'il n'atteint semble-t-il toute son efficacité que lorsqu'il est lu à voix haute.

9 Mes Amis, opus cit., p. 23. 
lecteur abasourdi de s'écrier : « Alors, c'est ça l'amour ! ».

L'amour, à l'ombre du père

Comment expliquer cet échec ? Les personnages masculins d'Emmanuel Bove n'ont pas, il est vrai, le charme ou l'ambition d'un Georges Duroy par exemple. Ce ne sont pas des séducteurs et leur situation est bien peu enviable la plupart du temps. Mais enfin, ils ne sont tout de même pas absolument repoussants : « J'aime à me regarder en face, à la lumière. Je me trouve mieux ${ }^{10} \gg$, déclare Victor Bâton dans une sorte de complaisance narcissique. L'échec des relations amoureuses tient selon moi essentiellement à un malaise filial. Une étude sur l'amour ne peut faire en ce sens l'économie d'une réflexion sur le thème de la filiation et, partant, sur celui de la famille en général. Le personnage bovien est un fils avant d'être un amant, comme l'illustrent notamment les nombreuses références à l'enfance traversant les textes de Bove. «Allons, allons mon petit. C'est vrai, vous êtes un enfant ${ }^{11}$ », déclare Alice à Nicolas Aftalion venu chercher un peu de réconfort.

Étudiant, au travers du sentiment de culpabilité notamment, l'aspect conflictuel des relations filiales dans l'œuvre bovienne, François Ouellet explique : «ce que le texte suggère, ce n'est pas une relation au père saisissable dans sa réalité, mais une relation imaginaire, une image du père qu'il a intériorisée et qu'il n'aura de cesse par la suite de symboliser dans ses relations amoureuses. ${ }^{12} \gg$ Le père en tant que personnage réel, est en effet physiquement absent des textes de Bove la plupart du temps ou, s'il apparaît, c'est généralement sous les traits du beau-père ou par le biais de la mémoire morcelée du fils coupable. Le plus souvent en outre, les personnages masculins ne connaissent pas leur père. Le narrateur des Mémoires d'un homme singulier, par exemple, est presque né de père inconnu. Quant au père de Nicolas Aftalion, il succombe dès les premiers temps du récit à une maladie respiratoire apparemment assez proche de celle qui emporta le père de Bove en $1915^{13}$. Mais la figure paternelle ne peut cependant être réduite à une représentation symbolique ou métaphorique. Le conflit relationnel ébranlant les rapports filiaux dans l'œuvre de Bove - et, indirectement, les rapports amoureux - résulte à notre sens avant tout d'un drame identitaire incontestablement lié à la mort réelle du père. Si elle pèse dès lors énormément sur l'œuvre de Bove - les

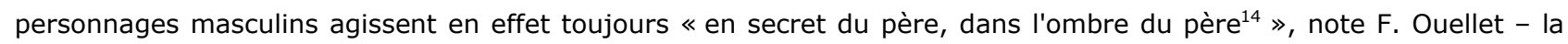
figure paternelle, destructurée par l'absence du père réel, n'en est pas moins fondamentalement défaillante. C'est ainsi que Alain Ferrant et René Roussillon faisaient remarquer à juste titre «combien la mort réelle du père désorganise profondément [,chez l'adolescent, le processus de construction des capacités de symbolisation et de l'identité de pensée]. Si le père imaginaire doit «mourir », l'assomption du père symbolique passe immanquablement par la « survivance » du père réel et quotidien. ${ }^{15}$ » La lecture d'un passage de La Coalition a justement attiré notre attention. Dans les dernières pages du roman, alors que les Aftalion semblent avoir touché le fond, Nicolas erre sans un sou dans

10 Ibid., p. 20.

11 La Coalition suivie de Un Raskolnikoff, Flammarion, 1986, p. 305.

12 François Ouellet, Emmanuel Bove. Contextes, références et écriture, préface de Bruno Curatolo, Québec, Nota Bene, 2005, pp. 172-173.

13 L'écrivain n'avait alors que 17 ans. Fait intéressant, Nicolas Aftalion n'a qu'une petite vingtaine d'années lorsque son père décède.

14 Emmanuel Bove. Contextes, références et écriture, opus cit., p. 171.

15 A. Ferrant et R. Roussillon, «Survivances du père et émergence du désir », Cahiers de psychologie clinique, n²4, 2005, pp. 167-184. 
le silence des rues parisiennes à peine éveillées. Bove écrit :

Il [...] apparut alors [à Nicolas] qu'il n'y avait pas au monde de douleur plus grande que la sienne. L'image de son père se présenta devant ses yeux. Il vivait encore, sans doute. Au cours des années troubles de son adolescence, il s'était longtemps imaginé que la mort de son père avait été inventée et qu'il se cachait en quelque pays lointain. Comme jadis, il se reprenait à douter. Il leva la tête, regarda le ciel où couraient de gros nuages noirs. Il eut alors la sensation de s'élever vers eux, [...]. Mais tout à coup il tomba ainsi qu'une pierre ${ }^{16}$.

Vraisemblablement, le personnage de La Coalition n'a pas fait le « deuil du père ». Il n'en a tout simplement pas eu le temps. Et ce raté dans l'étape décisive de la vie d'un jeune homme pourrait expliquer en partie son incapacité à vivre comme un adulte et à entretenir des relations sérieuses avec les femmes. Nous pensons en effet que l'échec de l'amour et du couple dans l'œuvre d'Emmanuel Bove est étroitement lié à la figure du «père mort ». Au meurtre symbolique ne succède aucunement la survivance du père réel. Après sa mort, d'ailleurs, Alexandre Aftalion ne laisse rien à Nicolas. Sans père, et sans repère, sans héritage donc, le jeune homme ne peut véritablement s'approprier ce qui le constitue ni s'inscrire pleinement, par conséquent, dans sa place de fils. Il ne peut en somme, selon les vers fameux du Faust de Goethe, posséder ce qui lui appartient ${ }^{17}$. Tragédie de la défiliation, de la déshérence, sans jeu de mot, condamnant la plupart des personnages masculins à l'errance. Incapable de toucher le père, incapable de l'atteindre, Nicolas semble s'élever vers lui pour retomber aussitôt dans la réalité. La présence paternelle est fantasmée, signe à la fois d'une terrible aporie identitaire et d'un appel à une partie de soi défaillante. Porteur d'un vide immense (le modèle paternel), doté d'un nom amputé (Bove ne s'appellerait-il pas Bobovnikoff ?), privé de sentiment social en somme, le personnage bovien reste pris dans une aboulie généralisée : «je voudrais pouvoir vouloir ${ }^{18} \gg$, déclare ainsi Louis Grandeville, le narrateur de Journal écrit en hiver (1931). « Je voudrais pouvoir aimer », semble-t-il avouer.

Finalement, que ce soit sous la forme d'un drame identitaire lié à la mort du père ou sous celle tout aussi traumatisante de la présence étouffante d'une mère possessive, l'ombre de la famille survole les récits boviens et régit les rapports amoureux. Si l'amour ne se résume finalement qu'à un idéal bien illusoire, c'est peut-être que tout ce qui le structurait et le légitimait jusqu'alors, à savoir le modèle familial, foyer par excellence des valeurs bourgeoises ${ }^{19}$, ainsi que la figure symbolique du père, s'effrite considérablement après-guerre. Les personnages boviens développent ainsi à la fois un irrépressible besoin de s'affranchir, psychologiquement et financièrement, des conventions dictées par la famille et l'incapacité de le faire réellement. C'est toute I'histoire du Beau-fils (1934) finalement, cet « embrouillamini conjugalo-familia ${ }^{20}$ » selon le mot de Claude Burgelin, et de L'Impossible amour également. Après une histoire pleine de rebondissements, durant laquelle l'ordre familial est sévèrement ébranlé, chaque chose finit par retrouver sa place. Les deux amants renouent avec leur père respectif, obtiennent leur bénédiction et rendent du même coup le mariage envisageable. Belle leçon de soumission à l'autorité paternelle qui laisse cependant à cette

16 La Coalition, opus cit., p. 317-318. Nous soulignons.

17 « Ce que tu as hérité de tes pères, il te faut l'acquérir, pour le posséder », Goethe, Faust, $1^{\text {ère }}$ partie, scène 1 , vers 682-683. Cité par Sigmund Freud dans Totem et Tabou (1912) et Moïse et le monothéisme (1938).

18 Emmanuel Bove, Journal écrit en hiver, Flammarion, 2006, p. 772.

19 Il faut préciser que la plupart des couples boviens ne sont pas mariés. Dans le cas contraire, cela ne dure pas en général. Je pense ici au couple du Pressentiment par exemple.

20 Claude Burgelin, «Un homme sans qualité. Lecture du Beau-Fils», in Lire Bove, présenté par Sophie Coste et Dominique Carlat, Presses Universitaires de Lyon, 2003, p. 234. 
union un goût bien amer. Pas d'alliance possible en effet où la communauté familiale n'impose pas ses lois. Rarement, chez Bove, l'amour peut se vivre librement hors des barrières étroites de la famille ou quand il y parvient, ce n'est que de manière temporaire, l'émancipation des amants n'aboutissant véritablement que dans la mort ou dans la solitude.

Le personnage bovien : un célibataire?

Une très grande partie du roman de l'échec durant l'entre-deux-guerres se donne ainsi à lire comme le lieu privilégié d'une reconfiguration œdipienne caractérisée par la triangularité et, simultanément, comme la tentative, souvent avortée, de son dépassement. La relation triangulaire est un thème récurrent dans ce que Philippe Chardin nomme à juste titre le roman de la conscience malheureuse : Svevo, Gorki, Proust, Mann, Musil, Martin du Gard, dont Bove est I'un des héritiers :

La période représentée dans les romans de la conscience malheureuse correspond [...] à un certain «âge d'or» de la névrose familiale et bourgeoise en Europe et [...] à la naissance de la théorie psychanalytique21.

L'amour du reste se vit souvent à trois chez Bove. Son œuvre regorge de personnages névrotiques, ces fils « incastrables » bien incapables de rechercher l'amour hors de l'éternel duo femme-inaccessible/homme-rival, mère interdite et père castrateur. Dans tous les cas, les relations amoureuses sont perturbées soit par un tiers séparateur, résurgence sans doute de la figure paternelle, venant s'immiscer dans les rapports amoureux et déclenchant un sentiment de jalousie intense, soit par le personnage lui-même qui, en proie à une pulsion narcissique, jette son dévolu sur un objet interdit rendu plus désirable encore par son inaccessibilité. Ainsi en est-il de Victor Bâton lorsque, pris dans un sentiment de jalousie envers Henri Billard qu'il considère pourtant comme son ami, il décide de séduire sa femme. Sur un plan purement psychologique, cela rend compte d'une réalité très instable du sentiment amoureux qui ressortit plus à l'égoïsme qu'à l'amour altruiste. Le personnage bovien est en constante recherche d'amour-propre. Il ne désire pas autrui pour ce qu'il est, mais par désir de son désir. Il désire être désiré en somme et n'a réellement le sentiment d'exister que s'il parvient à susciter un intérêt chez l'autre. Victor Bâton est un homme qui aspire sincèrement à aimer c'est certain. Mais il est également d'une incroyable mauvaise foi, conséquence logique d'un tropplein d'ipseité empêchant toute relation amoureuse : «Il est terrible de vivre ainsi sans être aimé, de toujours tout donner, de se dévouer, et de ne jamais rien recevoir en échange ${ }^{22} \gg$, déplore pitoyablement le narrateur de Journal écrit en hiver. Un détail, le personnage bovien ferme rarement les yeux lorsqu'il embrasse ses compagnes, ce qui pourrait trahir une incapacité à se donner, s'abandonner, se livrer pleinement. Le narrateur d'Armand déclare : « [Jeanne] s'approcha de moi, m'embrassa à plusieurs reprises. Je me vis contre son corps dans une glace. Elle croyait que j'avais fermé les yeux. ${ }^{23} \gg$

L'expérience sentimentale est en conséquence bien souvent décevante. Le fait de désirer posséder des femmes inaccessibles constitue paradoxalement la marque d'une véritable lucidité à l'égard des limites de toute

21 Philippe Chardin, Le roman de la conscience malheureuse, Genève, Droz, 1982, p. 187.

22 Journal écrit en hiver, opus cit., p. 763.

23 Emmanuel Bove, Armand, Flammarion, 2006, p. 139. 
relation amoureuse. «Malgré tout l'amour que j'ai pour Madeleine, déclare le narrateur de Journal écrit en hiver, elle n'est que la femme que j'ai rencontrée, alors que celle que j'aime je ne peux la rencontrer, car il est impossible qu'au milieu du monde je la trouve, parce qu'elle n'est peut-être pas née ou qu'elle est morte depuis des siècles. ${ }^{24} \gg$ Le personnage bovien comprend à ses dépens qu'un amour fusionnel avec la femme idéalisée, seul recours possible pour retrouver la jouissance et l'enfance perdue, n'existe pas. L'enfance de Bove, précisons-le, a été divisée entre deux femmes, sa mère et sa belle-mère ${ }^{25}$. L'image de la première était irrémédiablement ancrée à l'idée de misère, de souffrance et de frustration et celle de la seconde, sorte d'intermède à cette misère, logiquement associée au plaisir et à la satisfaction. Cette division douloureuse sera d'ailleurs déterminante dans l'œuvre de l'écrivain et influencera considérablement sa manière d'appréhender et de scinder la figure féminine, dans une sorte de prolongement métaphorique du clivage entre «bonne » et «mauvaise » mère, «bonne » et «mauvaise » compagne. Deux catégories stéréotypées de personnages féminins peuplent les romans de Bove. La femme réelle, calculatrice et pleine de minauderies et la femme idéale mais imaginaire. Le personnage bovien vit en somme dans le fantasme d'une vie de couple idéalisée. Il n'existe pleinement qu'au conditionnel, entre un passé familial assez peu édificateur et un avenir sentimental très incertain. «Ah ! Comme je voudrais être riche ! S'exclame Victor Bâton, [...] J'aurais une maîtresse, une actrice. [...] Je la recevrais dans une garçonnière au rez-de-chaussée d'une maison neuve. [...] J'embrasserai ses mains, son coude, ses épaules. Ensuite, ce serait l'amour ${ }^{26} \gg$.

Bâton et les autres personnages masculins multiplient à ce titre les remarques désobligeantes sur le caractère ou le physique de leurs maîtresses, comme si celles-ci, finalement, ne convenaient jamais. Bove lui-même écrit dans son journal : «J'ai connu beaucoup de femmes. Aucune ne me semble avoir été belle ${ }^{27}$ ». Le personnage bovien, comme son créateur, est un éternel insatisfait. Et de cette insatisfaction, à laquelle se greffe un sentiment de culpabilité durable, découle un comportement instable à l'égard de la femme aimée laissant transparaître il faut bien le reconnaître une certaine tendance à la misogynie (assez proche parfois de l'esprit traversant Les Jeunes filles de Montherlant). La plupart des personnages masculins peuplant le roman de l'échec ${ }^{28}$ sont à ce titre bien incapables d'accepter leurs compagnes telles qu'elles sont ou, s'ils y parviennent, ce n'est qu'après les avoir façonnées à leur guise selon un modèle idéal. Et cela, sous couvert de l'amour naturellement. Le narrateur de Journal écrit en hiver déclare :

Et ce qui m'est pénible, c'est que, quand j'essaye de la corriger, elle se fâche sans qu'une seconde elle distingue ce qu'il y a de vrai dans mes observations, de profondément amoureux dans le désir que j'ai qu'elle ne soit pas la risée de nos amis29.

Bien sûr, cette conception de la femme est l'expression d'une subjectivité blessée. Les personnages boviens développent une instabilité comportementale typique du schéma narcissique traversant le roman du début du siècle.

24 Journal écrit en hiver, opus cit., p. 764.

25 Voir à ce sujet la remarquable biographie de Jean-Luc Bitton et Raymond Cousse, Emmanuel Bove, La vie comme une ombre, Le Castor Astral, 1994.

26 Mes Amis, opus cit., pp. 26-28.

27 «Pages de journal (1936-1939) », in Emmanuel Bove, La vie comme une ombre, opus cit., p. 317.

28 Je pense notamment au Gueule d'Amour d'André Beucler et à certains personnages d'Eugène Dabit, Henri Calet ou Raymond Guérin.

29 Journal écrit en hiver, opus cit., p. 740. 
Louis le reconnaît lui-même : «c'est peut-être l'un des défauts que j'abomine le plus en moi que cette sorte d'emportement, de nervosité qui me fait porter des jugements, aimer ou détester quelqu'un pour, aussitôt après, émettre l'opinion contraire. ${ }^{30}$ » Évoquant la nature ambiguë des comportements amoureux dans le roman de la conscience malheureuse, Philippe Chardin parle de «loi du tout ou rien qui fait alterner euphorie mégalomaniaque et désespoir nihiliste... ${ }^{31} \gg$ Bove quant à lui ne fait-il pas dire à Nicolas Aftalion : «Il n'y a pas de milieu chez moi [...] tout ou rien ${ }^{32} \gg$ ? Pas de milieu non plus pour l'ensemble des personnages boviens qui oscillent constamment entre une tendresse apparemment sincère pour leur compagne et une forte suspicion générale à l'égard des femmes.

Derrière cet aspect duel de la figure féminine et cette situation paradoxale d'«amour dans la haine $\mathrm{d}^{33} \gg$, émerge cependant distinctement une image tout à fait positive et respectueuse des femmes. Il suffit de gratter un peu, c'est tout. Un rapide tour d'horizon des personnages féminins permet en effet de constater que la plupart d'entre eux fait preuve d'une lucidité et d'une force de caractère contrastant avec l'attitude aboulique et enfantine des protagonistes masculins. Ainsi en est-il par exemple de Colette, I'un des principaux personnages d'Un caractère de femme. Chez Bove, en effet, les rôles sont bien souvent inversés. Les femmes n'ont plus dans le couple la place qu'elles occupaient avant la guerre. Elles ne veulent plus être dominées et revendiquent notamment leur droit au plaisir. Les personnages masculins ont ainsi bien du mal à trouver leur place dans cette société bouleversée. Dans Mes Amis, Bâton rêve de recevoir sa maîtresse dans une garçonnière, mais quelques pages plus loin, c'est elle qui l'invite à monter dans sa chambre. Il avoue également frissonner comme un cheval sous les caresses de Lucie Dunois. La métaphore animale illustre bien la place centrale attribuée à la figure féminine. Chez Bove, ce sont les femmes, le plus souvent, qui tiennent les rênes.

Cette incapacité à aimer l'autre pour ce qu'il est réellement donne lieu à un inévitable rapport de force entre les deux amants. Dans Journal écrit en hiver, Madeleine finit par quitter Louis dont elle ne supporte plus les critiques, illustrant du même coup l'absence d'unité caractéristique des couples boviens. Il y a ainsi, suivant cette logique, une certaine prise de distance à l'égard de l'acte sexuel. Lorsqu'il apparaît, assez rarement il faut bien le dire, il est traité de manière burlesque ou présenté comme quelque chose d'interdit (là encore, les références à l'inceste et à l'adultère sont récurrentes). La sexualité est un aspect essentiel de l'atmosphère névrotique dans laquelle évolue la relation amoureuse. Autrui apparaît souvent comme un objet partiel. L'amour revêt alors une dimension profondément fétichiste et malsaine. Bâton focalise discrètement son regard sur les pieds et les chaussures de Blanche, tout comme Nicolas Aftalion sur ceux de sa future compagne. L'amour total, exclusif, la symbiose parfaite semblent bien illusoires. La figure féminine, finalement symbole d'altérité, est perçue de manière morcelée. Elle devient du même coup « étrangement inquiétante », non plus familière mais énigmatique, menaçante même pour l'intégrité narcissique des personnages masculins. Une double crainte empêche alors ces derniers de s'investir sereinement dans la relation amoureuse : la peur de l'étouffement ou de l'absorption de leur ego dans le couple et la peur de l'abandon. Mais un troisième facteur, pourtant, les pousse à rechercher la compagnie féminine : le poids de la solitude et la misère

30 Ibid., p. 772.

31 Philippe Chardin, Le roman de la conscience malheureuse, opus cit., p. 219.

32 La Coalition, opus cit., p. 234.

33 Formule empruntée à Dostoïevski, Les Carnets de I'Adolescent ; citée par Philippe Chardin, in L'amour dans la haine ou La jalousie dans la littérature moderne, Dostoïevski, James, Svevo, Proust, Musil, Droz, 1990, p. 33. 
existentielle dans laquelle ils végètent la plupart du temps. «Nec tecum nec sine te ${ }^{34} »$, telle serait finalement la formule résumant avec le plus de justesse la complexité des rapports humains et amoureux dans les œuvres d'Emmanuel Bove. «Je suis fait pour vivre seul, mais je ne puis rester seul35 », déclare le narrateur de Journal écrit en hiver.

Mal d'amour, mal de soi ?

C'est donc bien de cela qu'il s'agit : de l'éternelle incompréhension entre les êtres, de la complexité des rapports homme/femme, comme le montre ce passage de Journal écrit en hiver : «J'aime Madeleine, je suis jaloux d'elle, il me semble que ma vie serait terminée si elle venait à disparaître, et pourtant, en même temps, il y a quelque chose d'autre en moi. [...] J'aime Madeleine et en même temps je ne l'aime pas. ${ }^{36} \gg$ L'amour, la prétendue sincérité des sentiments, la comédie conjugale ne se résume finalement qu'à une vaine illusion conduisant forcément à une suite de malentendus, une série d'échec, une «succession d'abandons ${ }^{37}$ », selon le mot de Louis. Un rapprochement de I'univers bovien avec la conception proustienne de l'amour me paraît en ce sens évident. Les romans d'Emmanuel Bove, comme ceux de Proust, offrent une réalité conflictuelle et douloureuse du couple et de l'amour qui puise son pessimisme dans la thèse schopenhauerienne selon laquelle toute passion, et l'acte sexuel en particulier, reposerait sur une illusion. Cela expliquerait pourquoi, chez Bove notamment, le charme de la femme désirée cesse une fois l'objectif atteint. Pour l'auteur de La Recherche, le sentiment amoureux passe à travers le prisme de l'imaginaire artistique. Swann assimile par exemple Odette de Crécy, sa future épouse, à Zéphora, «qu'on voit dans une fresque de la chapelle Sixtine ${ }^{38} \gg$ peinte par Botticelli. Swann idéalise son amour. Il le crée de toutes pièces. De la même manière, quand ils n'assimilent pas l'objet de leur désir à une œuvre d'art - Florent Maugas, le héros de l'Impossible amour, compare ainsi Danièle Marondié, sa compagne, à La femme aux gants de Toulouse-Lautrec - les personnages boviens préfèrent jouer la comédie et feindre le bonheur à défaut de ne pouvoir l'atteindre réellement. «Ce qui me terrifie, déclare le narrateur de Journal écrit en hiver, c'est que je suis tout le temps malheureux et que tout le temps j'agis en homme heureux. ${ }^{39} \gg$ Le désenchantement auquel conduisent les sentiments voue irrémédiablement toute relation à l'échec. Or, ces échecs à répétitions provoquent une souffrance intense qui reviendrait ainsi à considérer l'amour comme une malédiction, une fatalité, une maladie. «Et cette maladie qu'était l'amour de Swann, déclare le narrateur de La Recherche, avait tellement multiplié, [...] il ne faisait tellement plus qu'un avec lui, qu'on aurait pas pu l'arracher de lui sans le détruire lui-même à peu près tout entier : comme on dit en chirurgie, son amour n'était plus opérable. ${ }^{40} \gg$ Même observation fataliste chez Bove. Ainsi Armand, qui vient de rompre avec Jeanne, explique : «j'eus soudain, dans le mouvement de la rue, pleinement conscience que tout était fini, que j'étais sans ressources, aussi

34 « Ni avec toi, ni sans toi », lettre de Samuel Beckett à Alan Schneider du 29 décembre 1957, reproduite partiellement dans Disjecta. Miscellaneous Writings and a dramatic Fragment by Samuel Beckett, éd. Ruby Cohn, Calder, 1983, p. 109. Nous reprenons ici un sous-titre de l'étude de Régis Salado, " "On n'est pas liés ?" Formes du lien dans En attendant Godot et Fin de partie », in Samuel Beckett, L'écriture et la scène, sous la direction de Evelyne Grossman et Régis Salado, Paris, SEDES, 1998, p. 95.

35 Journal écrit en hiver, opus cit., p. 757.

36 Ibid., p. 763.

37 Ibid., p. 760.

38 Marcel Proust, À la recherche temps perdu, tome 1, Du côté de chez Swann, France Loisir, 1999, pp. $245-247$.

39 Journal écrit en hiver, opus cit., p. 764.

40 Du côté de chez Swann, opus cit., p. 337. 
seul que devant une maladie. ${ }^{41} \gg$

Un dernier point, enfin, rapproche les deux écrivains. L'expérience amoureuse semble en effet pour eux intimement liée à la recherche de soi $^{42}$. La crise du couple annonce la crise identitaire qui touchera plus considérablement encore le personnage romanesque de l'immédiat-après-guerre. Analysant L'Amour de Pierre Neuhart et Un caractère de femme, Susanne Dürr faisait à juste titre remarquer que l'« une des questions les plus urgentes que le protagoniste moderne ait à affronter est de savoir qui il est. ${ }^{43}$ » Le thème de l'amour associé au mythe de Narcisse traduit en ce sens à la fois la volonté qu'ont les protagonistes masculins de se connaître pleinement et leur impossibilité de le faire. On l'a dit, le personnage bovien a développé depuis sa naissance ou son adolescence, à cause de la mort du père réel notamment, un vide immense qu'il cherche à combler par tous les moyens. En désirant posséder sa compagne, c'est lui qu'il souhaite conquérir. Peut-être espère-t-il ainsi reconstruire la part de son être qui l'empêche de trouver sa place ${ }^{44}$. Il n'y a rien en effet que l'amoureux ne désire en autrui que sa propre personne, car elle possède le double avantage de le mettre en présence de lui-même sans avoir à supporter le poids de la solitude. « J'aimerais mieux n'avoir personne autour de moi, déclare Louis Grandeville, n'être entouré que de silence, pour avoir seulement la certitude qu'il y a quelque part un être que j'aime et qui m'aime, un être pour lequel je suis tout. » Illusion de l'amour, désir d'exclusivité, fantasme de présence pleine et d'unité... L'expérience amoureuse, finalement, est peut-être avant tout le lieu où se joue le drame du sujet.

\section{Bibliographie}

\section{- Fuvres étudiées :}

Mes Amis, Flammarion, 2006.

Armand, Flammarion, 2006.

La Coalition suivie de Un Raskolnikoff, Flammarion, 1986.

Monsieur Thorpe et autres nouvelles, Le Castor Astral, 1988 et 2003.

L'Amour de Pierre Neuhart, Le Castor Astral, 1986.

Journal écrit en hiver, Flammarion, 2006.

Le Pressentiment, Le Castor Astral, 1991.

L'Impossible amour, Le Castor Astral, 1994.

41 Armand, opus cit., p. 212.

42 L'identité tient dans ce cas autant du nom que de la sexualité ; l'émergence, entre les deux guerres, de la « femme nouvelle » étant constitutif de ce malaise identitaire typiquement masculin.

43 Susanne Dürr, «Éléments d'une grammaire de la déchéance », in Lire Bove, opus cit., p. 203. Nous soulignons.

44 Les œuvres d'Emmanuel Bove sont emplies de personnages obsédés par le désir d'acquérir une situation. Ainsi en est-il de Jean-Marie Thély, le personnage principal largement autobiographique des Mémoires d'un homme singulier, lorsqu'il s'exclame : « Je n'ai rien demandé à l'existence d'extraordinaire. Je n'ai demandé qu'une seule chose. [...] C'est une place parmi les hommes, une place à moi, une place qu'ils reconnaîtraient comme mienne sans l'envier puisqu'elle n'aurait rien d'enviable. », pp. 25-26. Nous soulignons. Voir à ce sujet mon article sur « Le roman de l'échec dans l'entre-deux-guerres, l'exemple d'Emmanuel Bove », in Prix A'Doc de la jeune recherche en Franche-Comté, Presses Universitaires de Franche-Comté, 2008, pp. 83-92. 


\section{- Ouvrages sur Emmanuel Bove :}

BITTON, Jean-Luc et COUSSE, Raymond, Emmanuel Bove, la vie comme une ombre, Le Castor Astral, 1994.

BURGELIN, Claude, «Un homme sans qualité. Lecture du Beau-Fils », in Lire Bove, présenté par Sophie Coste et Dominique Carlat, Presses Universitaires de Lyon, 2003.

DÜRR, Susanne, «Éléments d'une grammaire de la déchéance », in Lire Bove, présenté par Sophie Coste et Dominique Carlat, Presses Universitaires de Lyon, 2003.

OUELLET, François, «La maîtrise implacable de l'écriture subjective», préface à l'édition de poche de Mes Amis, Québec, Nota Bene, 2002.

OUELLET, François, Emmanuel Bove, Contextes, références et écriture, préface de Bruno Curatolo, Québec, Nota Bene, 2005.

\section{- Ouvrages généraux :}

CHARDIN, Philippe, Le roman de la conscience malheureuse, Genève, Droz, 1982.

L'amour dans la haine ou La jalousie dans la littérature moderne, Dostö̈evski, James, Svevo, Proust, Musil, Droz, 1990.

FERRANT, A. et ROUSSILLON R., «Survivances du père et émergence du désir », Cahiers de psychologie clinique, $\mathrm{n}^{\circ}$ 24, 2005.

GROSSMAN, Evelyne et SALADO, Régis (dir.), Samuel Beckett, L'écriture et la scène, Paris, SEDES, 1998.

PROUST, Marcel, À la recherche temps perdu, tome 1, Du côté de chez Swann, France Loisir, 1999 